
A. Christe
R. Läubli
R. Guzman
U. Berlemann
R. J. Moore
G. Schroth
P. Vock
K. O. Lövblad

\section{Degeneration of the cervical disc: histology compared with radiography and magnetic resonance imaging}

Received: 15 January 2005

Accepted: 24 April 2005

Published online: 1 September 2005

(C) Springer-Verlag 2005

\author{
A. Christe $\cdot$ P. Vock \\ Department of Radiology, Inselspital, \\ University of Berne, Berne, Switzerland \\ R. Läubli · U. Berlemann \\ Department of Orthopaedic Surgery, \\ Inselspital, University of Berne, Berne, \\ Switzerland \\ R. Guzman · G. Schroth \\ K. O. Lövblad \\ Department of Neuroradiology, Inselspital, \\ University of Berne, Berne, Switzerland \\ R. J. Moore \\ Institute of Medical and Veterinary Science, \\ Adelaide, South Australia \\ K. O. Lövblad ( $\square)$ \\ Neuroradiology SRRI, Geneva University \\ Hospital, Rue Micheli-du-Crest 24, 1211 \\ Geneva 14, Switzerland \\ E-mail: karl-olof.lovblad@hcuge.ch \\ Tel.: + 41-22-3727033 \\ Fax: + 41-22-3727072
}

\begin{abstract}
Decisions about the treatment of neck pain are largely made on the basis of information gained from plain X-rays and magnetic resonance imaging (MRI), which are used routinely as part of preliminary investigation. We performed a descriptive cadaveric study to compare histology with radiography and MRI. We correlated plain radiography, disc height [Farfan index (FI)] and MRI findings with histology to assess the ability of radiology to detect significant pathologic lesions. The study included 52 motion segments from nine subjects over the age of 50, who underwent routine hospital autopsy. Disc degeneration was assessed by histology, radiography, disc height (FI: anterior disc height plus posterior disc height divided by anterioposterior diameter) and MRI using established grading systems. Most of the discs were classified radiologically as grade 1 $(19 / 52)$, grade $2(13 / 52)$, grade $3(9 /$ $52)$ or grade $4(3 / 52)$. Eight of the discs were graded as normal. The distribution of MRI grades was
\end{abstract}

grade $0(9 / 36)$, grade $1(9 / 36)$, grade $2(7 / 36)$, grade $3(8 / 36)$ and grade 4 $(3 / 36)$. Half of the discs $(26 / 52)$ showed advanced (grade 4) degeneration histologically. FI correlated with histological grade $(P=0.013)$, MRI grade $(P=0.02)$ and radiological grade $(P<0.001)$ of degeneration. Radiological and histological grade of degeneration showed a weak correlation $(r=0.3, P=0.033)$. MRI correlated with overall histological grade $(r=0.41, P=0.015$, $n=34$ ). Histological features (e.g., tears, rim lesions, prolapse of nucleus material) were poorly recognised by MRI, which had a sensitivity for disc material prolapse and annulus tears of less than $40 \%$. Our study showed that discs from patients over 50 years are histologically severely degenerated; however, these changes may not be detected by conventional radiography and MRI.

Keywords Cervical spine $\cdot$ Disc degeneration $\cdot$ Histology $\cdot$ Radiography $\cdot$ MRI $\cdot$ Cadaver study

\section{Introduction}

Back pain is a significant medical problem in western societies, reaching a peak in adults at around 4050 years of age. The prevalence of neck and shoulder pain is high-between 18 and $71 \%$ of the population. In addition, there is a higher incidence among manual laborers [1]. Autopsy studies have shown that by age 40, all individuals have some degenerative changes of the cervical spine [2].

Decisions about the treatment of neck pain are, to a large extent, made on the basis of information gained 
from plain X-rays and magnetic resonance imaging (MRI), which are used routinely as part of the preliminary investigation of affected patients. A few scientific studies have contributed to our knowledge by describing the normal histologic appearance of the lumbar spine from birth to senescence [4-6]. Furthermore, studies have shown the variety of pathologic conditions that could be responsible for pain symptoms, with a differential diagnosis encompassing prolapsed discs, disc degeneration, osteoarthrosis of the apophyseal joints, fractures and dislocations of vertebrae, osteoporosis, and spondylolisthesis [7]. We have not found any studies that have attempted to correlate histologic data with MRI and plain radiography. This is surprising given the high incidence of motor vehicle accident-associated whiplash injury and other causes of neck pain in western countries.

This study was undertaken firstly to describe the histological and radiological features, and grade of degeneration of the cervical discs in patients over 50 years. The second aim of the study was to correlate plain radiography, Farfan index (FI) and MRI findings with histology to assess the ability of radiology to detect significant pathologic lesions.

\section{Methods}

\section{Subjects}

The study included 54 motion segments from nine subjects over age 50, who underwent routine hospital autopsy. Cervical spine pain or previous surgery was not documented in the medical records of any of the subjects. Taking care to ensure that the musculature and ligaments were kept intact, the cervical spines were removed en bloc, sealed in double thickness polythene bags and frozen at $-20^{\circ} \mathrm{C}$.

\section{Radiography and MRI}

The specimens were thawed to room temperature before radiographic and MR imaging. Six of nine specimens underwent MRI. Anteroposterior and lateral X-rays were obtained from each specimen. The degree of disc
Table 2 Criteria for grading degenerative disc disease by MRI

\begin{tabular}{ll}
\hline & Grade \\
\hline Osteophytes and/or disc narrowing & $0 / 1$ \\
Disc prolapse & $0 / 1$ \\
Annular tears & $0 / 1$ \\
Decrease of signal intensity & $0 / 1$ \\
Sum & $0-4$ \\
\hline
\end{tabular}

degeneration was graded according to the classification system of Kellgren et al. [8, 9] (Table 1). Digitised images of the X-rays were used to calculate the FI [10] as a measure of relative disc height. The FI corresponds to a measure of relative disc height: the addition of anterior and posterior disc heights is divided by the anteroposterior disc diameter. MRI was performed on a $1.5 \mathrm{~T}$ Unit (Siemens Vision, Erlangen, Germany). The images were assessed in a blinded fashion by two radiologists and graded according to a classification system that described the features shown in Table 2. Each feature was given equal weighting and the grade assigned to the spine segment was an average value.

\section{Histology}

Immediately after X-ray and MRI, the motion segments were divided with a bandsaw by cutting the discs and facet joints into parasagittal slices approximately $5 \mathrm{~mm}$ thick. The blocks were photographed, fixed for $48 \mathrm{~h}$ in a solution with $4 \%$ formalin and 3\% Dextran and then immersed in a solution comprising 15\% EDTA and $0.5 \%$ paraformaldehyde until complete decalcification was confirmed by radiography. All slices were processed into paraffin wax using standard methods, and tissue sections of $5 \mathrm{~mm}$ thickness were stained by haematoxylin and eosin (H\&E) for histological examination. For each level, three disc sections representing the mid-, left- and right-parasagittal areas of the disc, were classified by two readers according to the grading system used in previous studies for histological changes in the lumbar spine [11] (Table 3). The grade given for each individual section was obtained by taking the worst characteristic feature observed; the overall grade for each level was the average of all sections analysed.

Table 1 Criteria for grading degenerative disc disease by radiography

\begin{tabular}{ll}
\hline Grade & Criteria \\
\hline 0 & Absence of degeneration in the disc \\
1 & Minimal anterior osteophytosis \\
2 & Definite anterior osteophytosis; possible narrowing of the disc space; some sclerosis of vertebral plates \\
3 & Moderate narrowing of the disc space; definite sclerosis of the vertebral plates; osteophytosis \\
4 & Severe narrowing of the disc space; sclerosis of the vertebral plates; multiple large osteophytes \\
\hline
\end{tabular}




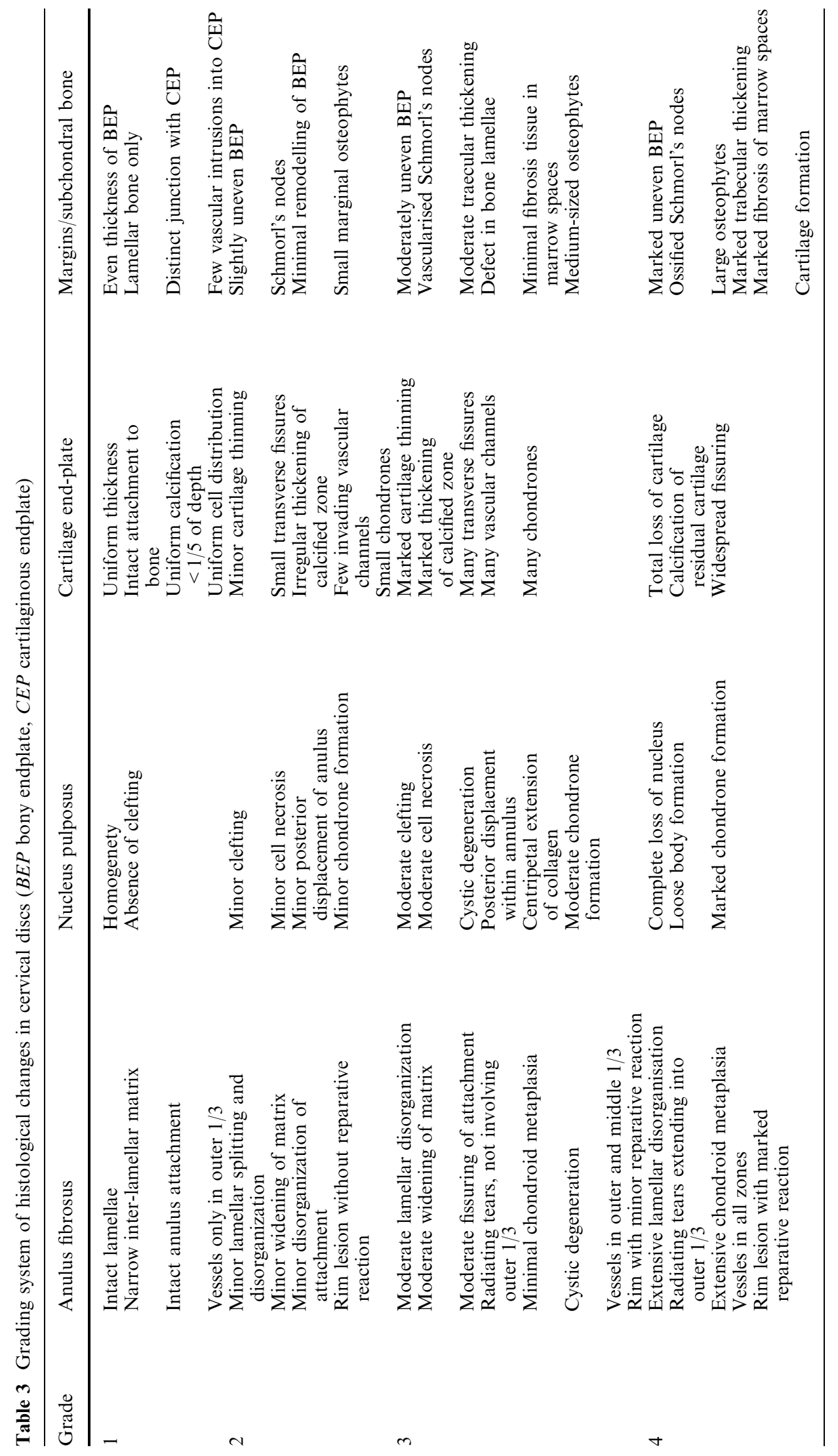




\section{Statistics}

The data were analysed using cross-tabulation, correlation analysis, paired $t$-tests (significance $P=0.05$ ) and frequency plots.

\section{Results}

\section{Plain X-ray}

Most of the discs were classified as grade $1(19 / 52)$, grade $2(13 / 52)$, grade $3(9 / 52)$ or grade $4(3 / 52)$. Eight of the discs were graded as normal. A total of $29 \%$ $(16 / 54)$ of the discs showed moderate to severe posterior, and $20 \%(11 / 54)$ showed moderate to severe

Fig. 1a-c Annular tear at $\mathrm{C} 3 / \mathrm{C} 4$ level. In this case the MRI shows irregularity of the posterior part of the disc (arrow). This is also seen on the histological HE section (arrow)

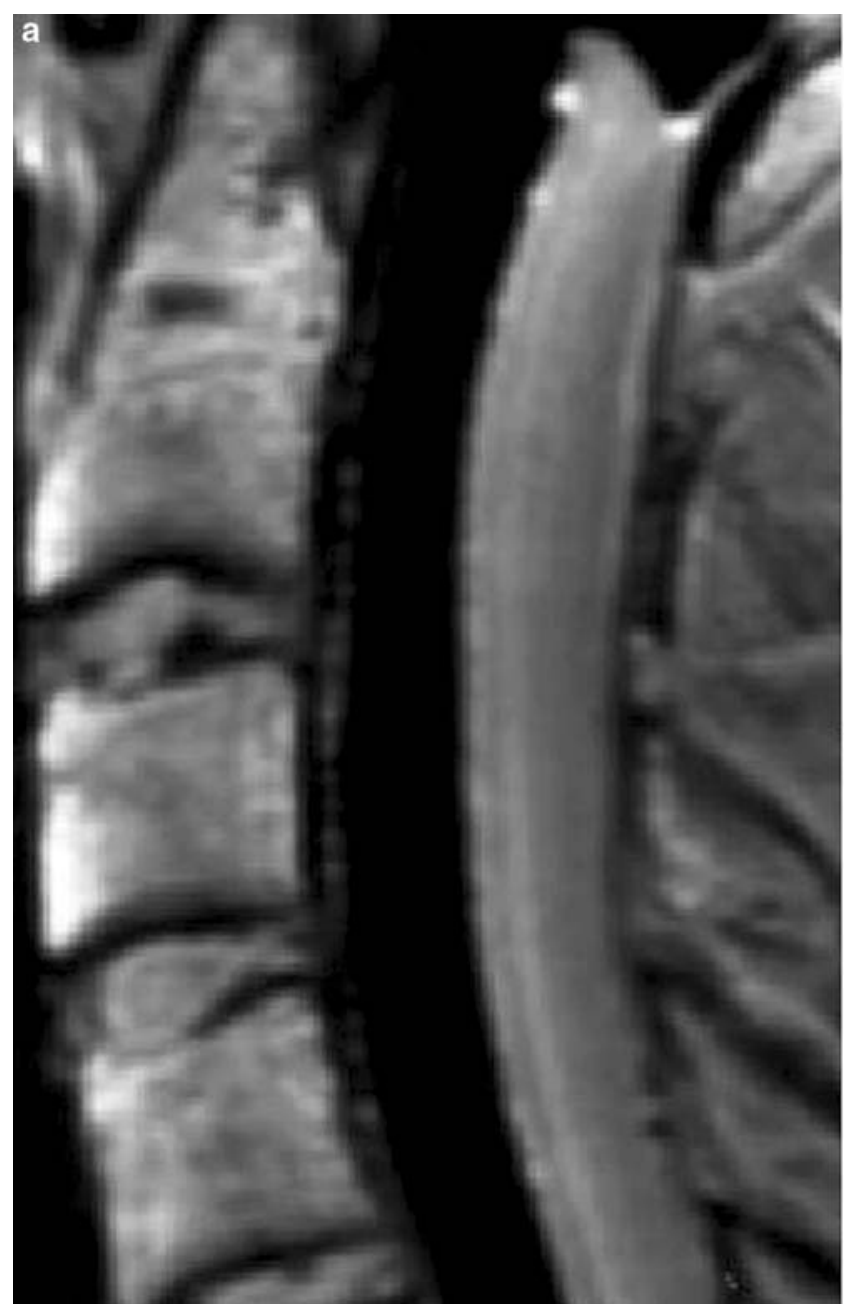

anterior osteophytosis. Moderate to severe sclerosis was noted in $26 \%(14 / 54)$ of the vertebral end plates, and $15 \%(8 / 54)$ of the disc spaces were moderately to severely narrowed (Table 1). The FI tended to be lowest at level 5/6.

\section{Magnetic resonance imaging}

The distribution of MRI grades was: grade $0(10 / 36)$, grade $1(8 / 36)$, grade $2(7 / 36)$, grade $3(8 / 36)$ and grade 4 $(3 / 34)$ with a trend towards more advanced pathology in the discs at C4-5 and C5-6. Radiating tears (Fig. 1) were observed in $40 \%(14 / 36)$ of the discs, bulging prolapse in $31 \%(11 / 36)$ (Figs. 2 and 3), osteophytosis and disc space narrowing in $42 \%(15 / 36)$ and loss of signal intensity in 53\% (19/36).

\section{Histology}

Half of the discs (26/52) showed advanced (grade 4) degeneration. Radiating tears of the annulus were

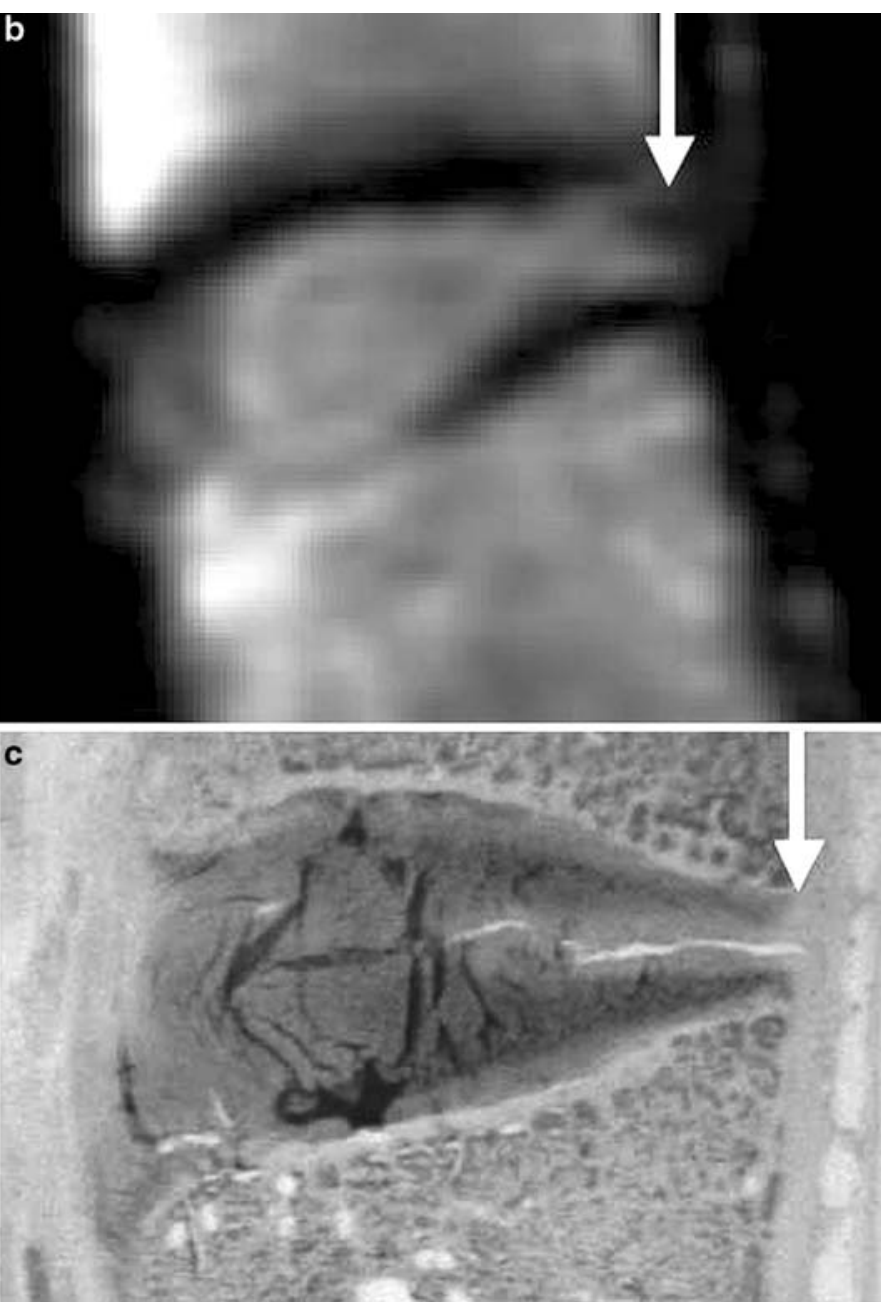




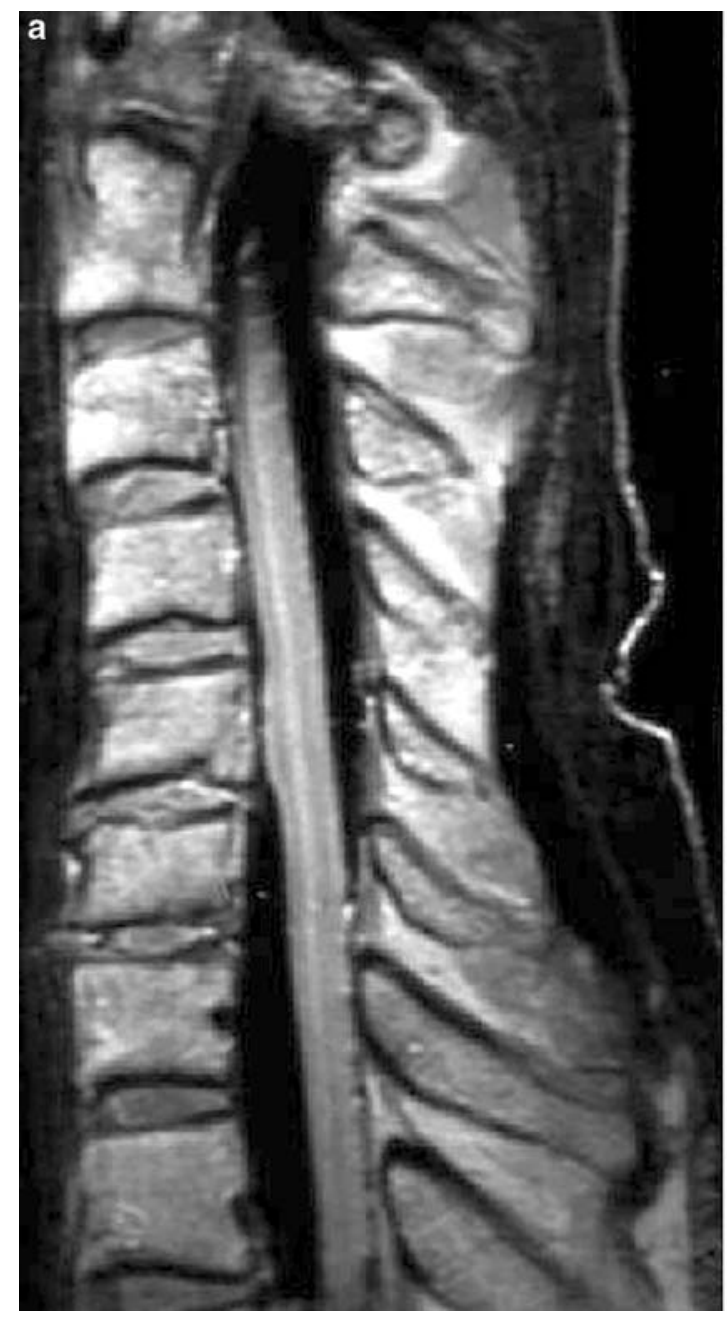

Fig. 2a-c Disc prolapse at $\mathrm{C} 4 / 5$ level only seen on histological HE section (arrow) but not on MRI

common and in most cases extended to the peripheral one-third of the discs $(64 \%, 34 / 52)$. Lesions were more common in the posterior annulus (12 discs) than in the anterior annulus (three discs), but tears extending from the front to the back of the discs were most common (27 discs). We observed rim lesions of the annulus in $64 \%(34 / 52)$ of the discs, and half of the cases were accompanied by some vascularised granulation tissue. Anterior rim lesions were more common (20 discs) than posterior lesions (nine discs). We found some prolapse of nucleus material either anterior (four discs), posterior (12 discs) or anterior and posterior simultaneously (22 discs) in $74 \%(38 / 52)$ of the discs. In four cases there was disc material between the posterior anulus and the ligament, but without rupture of the annulus. In $52 \%(28 / 52)$ of the discs, remodelling of the bony margins resulted in the formation of osteophytes, approximately half of which were graded

\section{Correlations}

Subjective assessment of disc narrowing on plain radiographs was confirmed by the FI of relative disc height $(P<0.001)$. The FI also correlated with histological grade $(P=0.013)$, MRI grade $(P=0.02)$ and radiological grade $(P<0.001)$ of degeneration. Radiological and histological grade of degeneration showed a weak correlation $(r=0.3, P=0.033)$ (Fig. 4). The sensitivity of the radiography to detect osteophytes was $95 \%$ for anterior and $92 \%$ for posterior osteophytes. The specificity was $100 \%$. In $39 \%$ (14/34) of the cases where we observed some prolapse of nucleus material, disc space narrowing was detected in the radiography. There was no correlation between prolapse of nucleus material and the FI. MRI correlated with overall histological grade $(r=0.41, P=0.015, n=34)$. MRI detected $62 \%$ $(18 / 28)$ of the osteophytes, $38 \%(10 / 27)$ of nucleus 

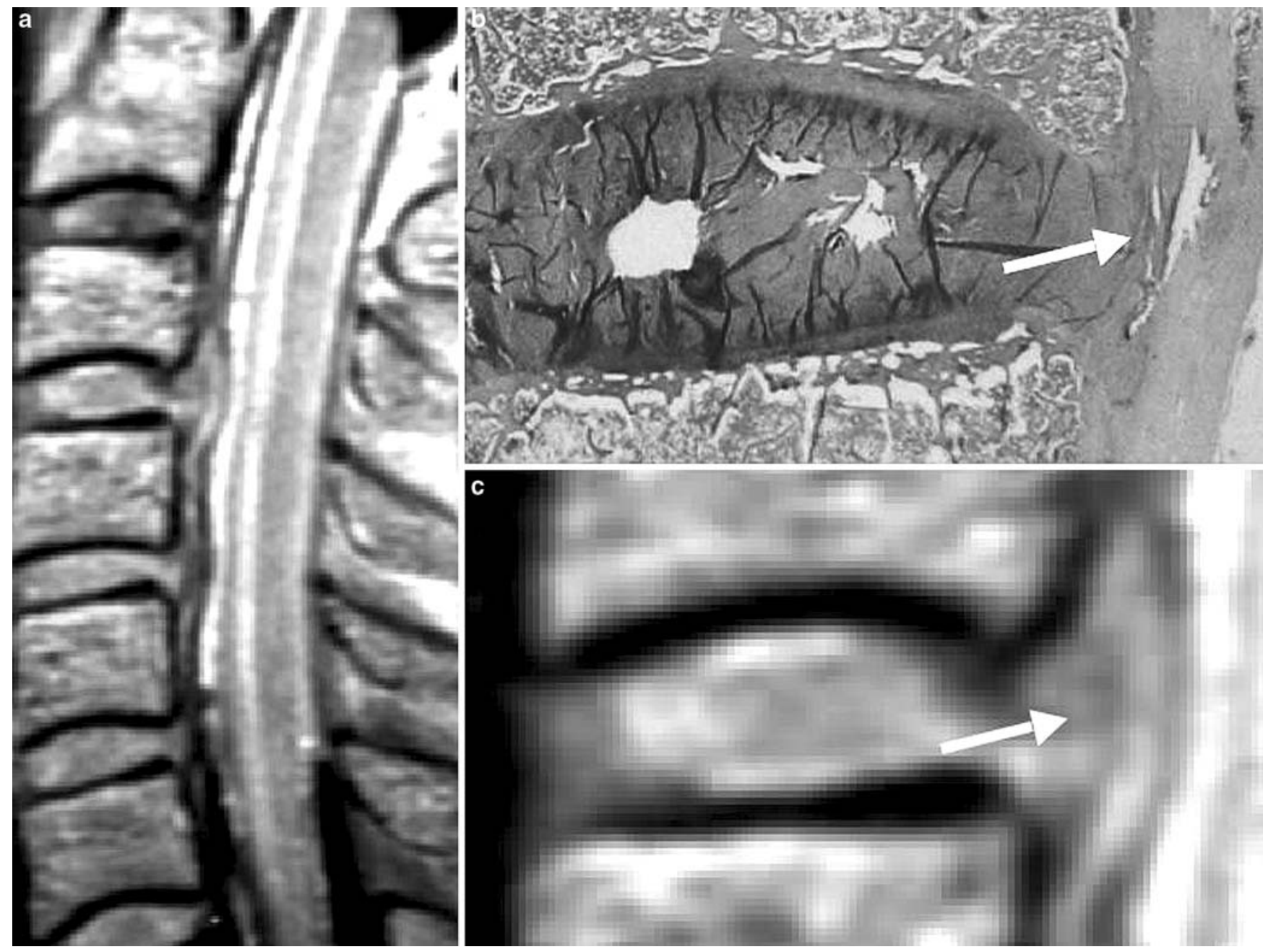

Fig. 3a-c Disc prolapse at C3/4 level only seen on histological HE section (arrow) and on MRI

Fig. 4 Correlation between radiological findings and histopathology prolapse and $37 \%(8 / 22)$ of the radiating tears. MRI grade of degeneration correlated with radiologic grade of degeneration $(r=0.43, P=0.01, n=34)$. Table 4 shows the correlation between histological and MRI

\section{Histologic Grade versus Radiologic Grade}

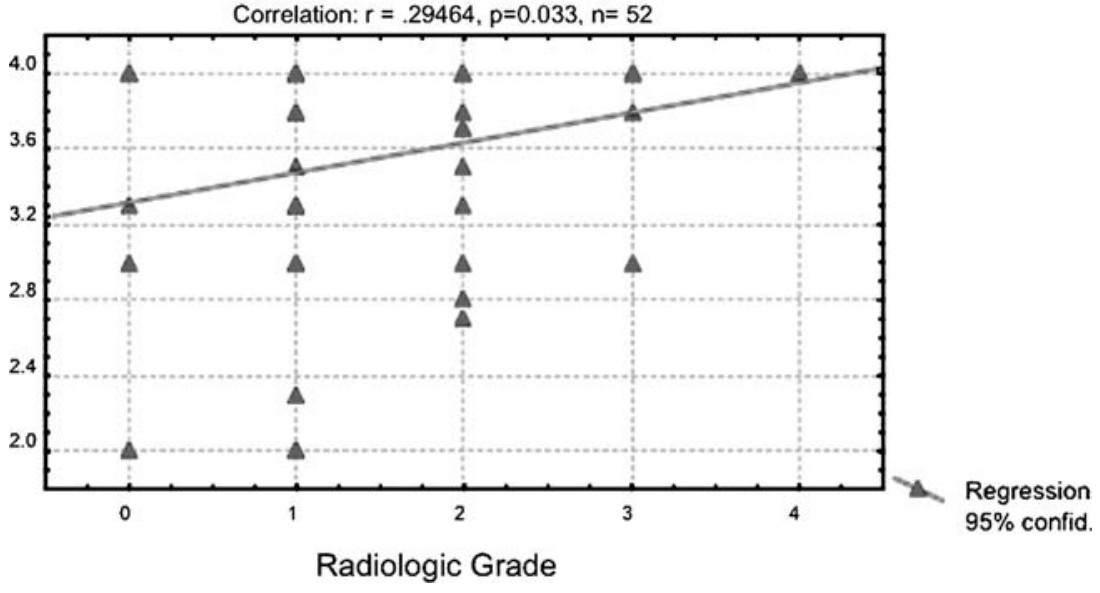


Table 4 A comparison of MRI and histologic findings

\begin{tabular}{ll}
\hline MRI appearance of disc on T2 images & \\
\hline Histologic finding & Correlation coefficient $(r)$ \\
\hline Annular tears & -0.14 \\
Osteophytes & $0.46^{*}$ \\
Nucleus prolapse & 0.14
\end{tabular}

*Significant correlation, $P<0.05$

findings (Fig. 5). Age did not correlate with the grade of disc degeneration.

\section{Discussion}

In this study we have shown that discs from patients over 50 years of age are histologically severely degenerated; however, these changes may not be detected by conventional radiography and MRI.

This represents a problem, since MRI is typically the method of choice for non-invasive investigation of the spine and spinal canal in patients with pain. Histology is a reliable and sensitive method that has been used to show the natural sequence of degeneration in the human spine. While histology may be the best method to see the structural detail of the spine components, it is obviously not applicable to clinical investigations. For this reason, non-invasive imaging techniques such as plain radiography and MRI are used for initial investigation of patients with spinal disorders. In light of recent knowledge it is appropriate to investigate the ability of these techniques to detect reliably the structural changes to spinal components.

The key question is whether these changes can be seen or predicted radiologically. Indeed, we found correlations between the histologic grade of degeneration and radiography, FI and MRI. However, we did not find a significant correlation between MRI signs of annular tears and histologic annular tears, indicating that MRI is not reliably able to detect this lesion. MRI could not demonstrate full thickness radiating annular tears in any of the discs. These tears are associated with nuclear prolapse and might be expected to elicit pain symptoms from the release of inflammatory mediators [12-14]. Overall, the sensitivity of the MRI to detect tears and nucleus prolapse was less than $40 \%$; thus, our study did not confirm the results from $\mathrm{Yu}$ et al. [15], which suggested that MR imaging was an accurate diagnostic tool for investigating tears of the anulus. Two previous studies which compared MRI with discography in the lumbar spine also found that significant annular tears are often missed by MRI [16-18]. In our study, the discs showed no significant loss of T2-weighted signal, and there was no significant loss of disc height, which may be a feature that is peculiar to cervical discs. In addition, the correlation in our study of histology with the FI and radiography demonstrates this. However, when comparing previous studies to our series, it should be noted that degenerated lumbar discs are significantly narrower than cervical discs $[11,19,20]$. In half of the cases we observed anterior nucleus prolapse, a feature which is rarely noted in routine MRI examinations, although it could result in an irritation of nerve endings in the ligamentum longitudinale anterior or anulus fibrosus and thus elicit pain [21-24].

In this study, MR was a moderate indicator of advanced disc pathology (grade 4) only and poor at detecting lesser degrees of pathology that were confirmed by microscopy. This is also supported by a macroanatomic study by Vikarii et al. [25], who reported that MRI correlated weakly with macroanatomy of disc degeneration and that nuclear intensity changes tended to underestimate such changes. In our study, four were classified by MRI as healthy discs (grade 0), which
Fig. 5 Correlation between MRI findings and histopathology

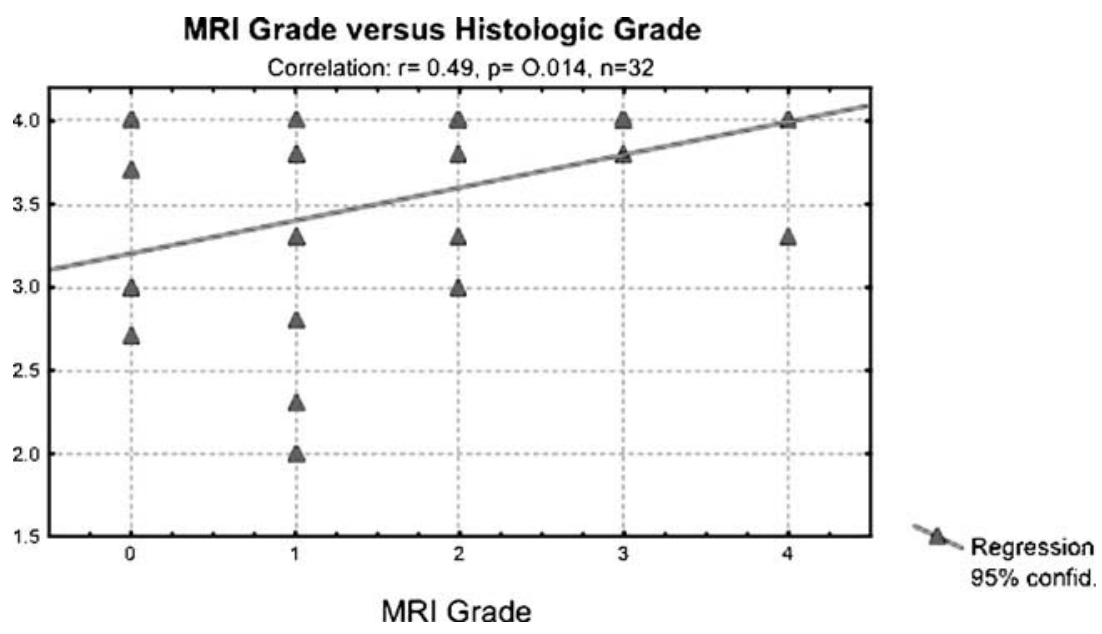


corresponded to two out of 16 discs that were graded histologically as normal.

Typically, grade 4 discs were severely degenerated with extensive annular tearing, with either partial or complete nuclear prolapse. MRI would not be a critical investigation in such cases as patients would generally present with symptoms that would be detected during careful clinical examination. And, as our study revealed, disc protrusion (bulging) on MRI is in most cases histologically a prolapse, since MRI cannot recognise earlier stages of disease. A previous study showed that the interobserver reliability for the diagnosis of a disc protrusion was only moderate [26]. Previous studies have also shown that reduced T2weighted signals on MRI correlate with decreased water content and proteoglycan concentration in lumbar discs, as well as general radiologic indicators of disc degeneration [27-31]. As in our study, these studies found that there was no correlation between MRI and histologic features of early degeneration. In terms of the treatment of degenerative spinal disorders, it is probably more important for MRI to detect these early changes. The failure of MRI in this study to detect early changes probably reflects the inherent limitations of the technique and may be overcome with improved resolution of newer devices.

Our results call into question the study of Dai [31], which confirms cervical segmental instability as an indicator for early degeneration; importantly, we may have to redefine the expression "early" since MRI may not be able to recognise histologically confirmed early degeneration [32]. Indeed, Dai et al. found a correlation between MRI findings on T2-weighted images with disc degeneration and findings on cervical spine flexion radiographs, indicating that MRI could detect early changes. However, they did not perform any histological studies, as we did, and, therefore, did not have pathological confirmation. In our study, MRI was often unable to demonstrate early histologic changes.

To our knowledge, no other studies comparing histology to radiology of the cervical spine have been performed as extensively as those concerning the lumbar spine. We have extrapolated our findings to those from lumbar spine studies; this represents one of the weaknesses of this paper, since there are well-know biomechanical differences between these two spinal segments.

Spinal degeneration is an inevitable consequence of aging. While it is not possible to stop this degenerative process, understanding the basis for such changes may provide options for the treatment of the pain and disability associated with aging. If it is possible to demonstrate reliably particular morphologic features that are associated with painful discs and facet joints, it may increase therapeutic options for the clinical treatment of degenerative spinal disorders. Clinicians have to be careful when interpreting X-ray and MRI, because discs which seem to be normal on X-ray and MRI can have degenerative changes which could induce pain (false negative results). Conversely, there is no proof that highly degenerated discs are a source of pain (false positive results) [27]. Although one of the aims of this study was to provide data on the normal processes involved with cervical motion segment degeneration, we were only able to provide data on specimens that were well advanced in the degenerative process due to difficulties in specimen collection.

\section{References}

1. Andersson HI (1994) The epidemiology of chronic pain in a Swedish rural area. Qual Life Res 3 (Suppl 1):S19-S26

2. Nathan H (1962) Osteophytes of the vertebral column. An anatomical study of their development according to age, race, and sex, with consideration as to their etiology and significance. $\mathrm{J}$ Bone Joint Surg Am 44:243-268

3. Lebkowski WJ (2002) Ageing and degeneration of human lumbar intervertebral discs. Pol Merkuriusz Lek 13:234-237

4. Gries NC, Berlemann U, Moore RJ, Vernon-Roberts B (2000) Early histologic changes in lower lumbar discs and facet joints and their correlation. Eur Spine J 9(1):23-29
5. Yoshida M (1969) Histopathological and histochemical studies on age-related changes in the intervertebral discs, regional ligaments and vascular systems of the lumbar vertebrae. Nippon Ika Daigaku Zasshi 36:61-80

6. Ritchie JH, Fahrni WH (1970) Age changes in lumbar intervertebral discs. Can J Surg 13:65-71

7. Kelsey JL, White AA III (1980) Epidemiology and impact of low-back pain. Spine 5:133-142

8. Kellgren J, Jeffrey MR, Ball J (eds) (1963) The epidemiology of chronic rheumatism. In: Atlas of standard radiographs. Blackwell Scientific, Oxford, pp 14-19
9. Coté P, Cassidy D, Young-Hing K (1996) Apophysial joint degeneration, disc degeneration, and sagittal curve of the cervical spine. Spine 22:859-864

10. Farfan HF, Huberdeau RM, Dubow HI (1972) Lumbar intervertebral disc degeneration: the influence of geometrical features on the pattern of disc degeneration - a post-mortem study. J Bone Joint Surg Am 54:492-510

11. Berlemann U, Gries NC, Moore RJ (1998) The relationship between height, shape and histological changes in early degeneration of the lower lumbar discs. Eur Spine J 7:212-217 
12. Osti OL, Vernon-Roberts B, Moore R, Fraser RD (1992) Annular tears and disc degeneration in the lumbar spine. A post-mortem study of 135 discs. J Bone Joint Surg Br 74:678-682

13. Osti OL, Vernon-Roberts B, Fraser RD (1990) 1990 Volvo Award in experimental studies. Anulus tears and intervertebral disc degeneration. An experimental study using an animal model. Spine 15:762-767

14. Cloward RB (1952) Discography: technique, indications and evaluation of normal and abnormal intervertebral disc. AJR Am J Roentgenol 68:552-564

15. Yu SW, Sether LA, Ho PS, Wagner M, Haughton VM (1988) Tears of the anulus fibrosus: correlation between MR and pathologic findings in cadavers. AJNR Am J Neuroradiol 9:367-370

16. Gunzburg R, Parkinson R (1991) A cadaveric study comparing discography, MRI, histology, and mechanical behavior of the human lumbar disc. Spine 17:417-423

17. Kaiser JA, Holland BA (1998) Imaging of the cervical spine. Spine 23:27012712

18. Schellhas KP (1996) Cervical discogenic pain. Prospective correlation of magnetic resonance imaging and discography in asymptomatic subjects and pain sufferers. Spine 21:300-311
19. Vernon-Roberts B, Pirie CJ (1977) Degenerative changes in the intervertebral discs of the lumbar spine and their sequelae. Rheumatol Rehabil 16:13-21

20. Amonoo-Kuofi HS (1991) Morphometric changes in the heights and anteroposterior diameters of the lumbar intervertebral discs with age. $\mathbf{J}$ Anat 175:159-168

21. Coppes MH, Marani E, Thomeer RT, Groen GJ (1997) Innervation of "painful" lumbar discs. Spine 22:2342-2349

22. Hirsch C (1963) The anatomical basis for low back pain. Acta Orthop Scand 33:1-17

23. Kuslich SD (1991) The tissue origin of low back pain and sciatica: a report of pain response to tissue stimulation during operation on the lumbar spine using local anesthesia. Orthop Clin North Am 22:181-187

24. Imai S, Konttinen YT, Tokunaga Y, Maeda T, Hukuda S, Santavirta S (1997) An ultrastructural study of calcitonin gene-related peptide-immunoreactive nerve fibers innervating the rat posterior longitudinal ligament. A morphologic basis for their possible efferent actions. Spine 22:1941-1947

25. Viikari-Juntura E, Raininko R, Videman T, Porkka L (1989) Evaluation of cervical disc degeneration with ultralow field MRI and discography. An experimental study on cadavers. Spine 14:616-619

26. Matsumoto M, Fujimura Y, Suzuki N et al (1998) MRI of cervical intervertebral discs in asymptomatic subjects. J Bone Joint Surg 80:19-24
27. Schiebler ML, Camerino VJ, Fallon MD, Zlatkin MB, Grenier N, Kressel HY (1991) In vivo and ex vivo magnetic resonance imaging evaluation of early disc degeneration with histopathologic correlation. Spine 16:635-640

28. Paajanen H, Komu M, Lehto I, Laato M, Haapasalo H (1994) Magnetization transfer imaging of lumbar disc degeneration. Correlation of relaxation parameters with biochemistry. Spine 19:2833-2837

29. Pearce RH (1991) Magnetic resonance imaging reflects the chemical changes of aging degeneration in the human intervertebral disc. J Rheumatol 18:42-43

30. Tertti M, Paajanen H, Laato M, Aho H, Komu M, Kormanc M (1991) Disc degeneration in magnetic resonance imaging. A comparative biochemical, histologic, and radiologic study in cadaver spines. Spine 16:629-634

31. Dai L (1998) Disc degeneration and cervical instability. Correlation of magnetic resonance imaging with radiography. Spine 23:1734-1738

32. Boden SD, McCowin PR, Davis DO, Dina TS, Mark AS, Wiesel S (1990) Abnormal magnetic-resonance scans of the cervical spine in asymptomatic subjects. A prospective investigation. J Bone Joint Surg 72:1178-1184 